\title{
Produção de aflatoxinas e ácido ciclopiazônico por cepas de Aspergillus flavus isoladas de amendoim
}

\author{
Production of aflatoxin and cyclopiazonic acid by Aspergillus flavus strains \\ isolate from peanuts
}

\section{Edlayne Gonçalez ${ }^{1 *}$, Janaina Lara da Silva', Tatiana Alves dos Reis ${ }^{2}$, Viviane Kobushi Nakai Joana D'Arc Felicio', Benedito Corrêa²}

| | | | | | | | | | | | | | | | | | | | | | | | | | | | | | | | | | | | | | | | | | | | | | | | | | | | | | | | | | | | | | | | | | | | | | | | | | | | | | | | | | | | | | | | | | | | | | | | | | | | | | | | | | | | | | | | | | | | | | | | | | | | | | | | | | | | | | | | | | | | | | | | | | | | | | | | | | | | | | | | | | | | | | | | | | | | | | | | | | | | | | | | | | | | | | | |

RESUMO: Aspergillus flavus é um fungo filamentoso que pode produzir aflatoxinas e ácido ciclopiazônico, sendo que a presença dessas micotoxinas em alimentos e raçóes pode levar a um efeito tóxico no homem e em animais. Cepas de $A$. flavus produtoras de aflatoxinas e ácido ciclopiazônico são frequentemente isoladas do amendoim, indicando a natural co-ocorrência dessas toxinas neste substrato. Neste estudo, foram isoladas 47 cepas de Aspergillus flavus em grãos e cascas de amendoim durante diferentes fases de maturação do fruto e também durante seu armazenamento. Das cepas isoladas, foram avaliados os potenciais para produção de aflatoxinas e ácido ciclopiazônico, em que 91,5\% foram produtoras de aflatoxinas e $70 \%$ produziram ácido ciclopiazônico, sendo que $63,8 \%$ produziram ambas as toxinas e $2,1 \%$ não produziu nenhuma. A presença de cepas toxigênicas de A. flavus nas amostras de amendoim analisadas indica um risco potencial da contaminação deste produto, caso seja exposto a condiçóes ambientais favoráveis ao crescimento do fungo e produçáo de micotoxinas.

PALAVRAS-CHAVE: micotoxinas; fungos toxigênico; produção de micotoxinas; ocorrência.

\begin{abstract}
Aspergillus flavus is a filamentous fungus that can produce aflatoxins and cyclopiazonic acid, and the presence of these mycotoxins in food and feed can lead to a toxic effect on humans and animals. Strains of $A$. flavus producing aflatoxin and cyclopiazonic acid are often isolated from peanuts, indicating the the co-occurrence of these toxins in the natural substrate. In this study, we isolated 47 strains of A. flavus on peanut kernels and hulls during different stages of fruit ripening and its storage. Of the isolated strains, we assessed the potential for aflatoxin and cyclopiazonic acid production, in which $91.5 \%$ could produce aflatoxins and $70 \%$ produced cyclopiazonic acid; $63.8 \%$ produced both toxins and $2.1 \%$ produced no toxin. The presence of toxigenic $A$. flavus strains in peanut samples indicate a potential risk of contamination of these products, if they are exposed to environmental conditions that are favorable to fungal growth and mycotoxin production.
\end{abstract}

KEYWORDS: mycotoxins; toxigenic fungi; mycotoxin production; occcurrence. 


\section{INTRODUÇÃO}

Os fungos podem causar uma série de danos aos grãos durante o plantio e a colheita, bem como durante o armazenamento (Sillker; Elliot, 1980), sendo que Aspergillus, Fusarium e Penicillium são os principais gêneros de fungos que produzem micotoxinas em alimentos (Siame et al., 1998). Micotoxinas são substâncias tóxicas produzidas pelo metabolismo secundário de várias espécies de fungos filamentosos, os quais contaminam alimentos no campo, no armazenamento ou após a manufatura (Moss, 1996). Os fungos toxigênicos são conhecidos por produzir uma ou mais micotoxinas (Hussein, 2001). Aspergillus flavus é um fungo filamentoso que pode produzir aflatoxinas e ácido ciclopiazônico, sendo que a coprodução dessas micotoxinas pode resultar em efeito tóxico aditivo ou sinérgico nos consumidores, aumentando o potencial toxigênico deste fungo (GQAlEni et al., 1997). Em geral, amendoim, milho e sementes de algodão estão entre os grãos com maior risco de contaminação (IsmaIL, 2000). No Brasil, a contaminação de amendoins por aflatoxinas é um grande problema devido a fatores ambientais que favorecem o crescimento de fungos e a produção da toxina (CALORI-Domingues; FonseCA, 1995). O ácido ciclopiazônico ocorre naturalmente no milho e no amendoim juntamente com as aflatoxinas (VAAMONDE et al., 2003).

Vários estudos foram conduzidos para identificar a microbiota fúngica e as micotoxinas no amendoim durante a estocagem e em condiçóes de campo experimentais, sendo que os principais gêneros encontrados foram Aspergillus, Penicillium e Fusarium, e entre as micotoxinas, as aflatoxinas e o ácido ciclopiazônico (Lisker et al., 1993; Horn et al., 1994; 1995; 2000; ANDERSON et al., 1996; SiAme et al., 1998; 2000; Ismail, 2000; BHATTACHARYA; RAHA, 2002; VAAMONDE et al., 2006; Mphane et al., 2004; Pildain et al., 2004; GonçAlez et al., 2008a, 2008b; NaKai et al., 2008; Zorzete et al., 2011, 2013; Atayde et al., 2012). BAQUIÃo et al. (2012) relataram alta frequência de $A$. flavus toxigênicos em solo no qual são coletadas castanhas do Brasil na Amazônia, assim como a presença destes nos frutos e casca.

A contaminaçáo por aflatoxinas é o principal problema de segurança alimentar nas culturas de campo, particularmente de itens que são produzidos em climas tropicais e sutropicais em países nos quais a alta temperatura e a umidade promovem o crescimento e a proliferação de Aspergillus spp. (RudREw et al., 2013).

Este trabalho teve como objetivo isolar cepas de Aspergillus flavus das cascas e grãos de amendoim durante as diferentes fases de maturação do fruto durante um ano de armazenamento, verificando o potencial toxigênico destas quanto à produçáo de aflatoxinas $\mathrm{B}_{1}$ e $\mathrm{B}_{2}$ e o ácido ciclopiazônico.

\section{MATERIAL E MÉTODOS}

\section{Local de coleta}

O estudo foi realizado em Junqueirópolis, Estado de São Paulo, Brasil. A semente de amendoim utilizada no plantio foi da variedade Runner IAC 886, plantada no mês de dezembro de 2004. O amendoim foi colhido em março de 2005 e armazenado por um ano. Foram realizadas quatro coletas no campo de amendoim nas seguintes fases de maturação: fruto recém-formado, em início de granação, em granação, maduro e após a secagem. Os frutos foram armazenados por um ano, de junho de 2005 a junho de 2006. Foram realizadas coletas mensais em triplicata.

\section{Isolamento e identificação dos fungos}

De cada uma das 25 amostras de amendoim foram retiradas, aproximadamente, $30 \mathrm{~g}$ para desinfecção em solução de hipoclorito de sódio $2 \%$ por 3 minutos. Após a desinfecção, separou-se a casca dos grãos do amendoim, que foram lavados com água estéril. Foram utilizadas, para cada amostra em meio de cultura, 3 placas de Petri contendo 11 grãos cada e 3 placas contendo cascas semeadas diretamente em meio Ágar DRBC e AFPA (Piтt et al., 1979). Todas as placas foram incubadas a $25^{\circ} \mathrm{C}$ por cinco dias e os resultados foram expressos em porcentagem do total de grãos inoculados contaminados por fungos. Em seguida, colônias de diferentes tipos morfológicos foram isoladas em Ágar Sabouraud Dextrose e submetidas à identificação por meio da técnica de microcultivo (RidDELL, 1950). Os fungos pertencentes ao gênero Aspergillus foram classificados por espécie de acordo com os seguintes compêndios: ARX (1974), Barnett; Hunter (1965), Barron (1972), Ellis (1993), Nelson et al. (1983), Raper; Fennel (1965).

\section{Produção de Micotoxinas Aflatoxinas}

As cepas de Aspergillus spp. isoladas e identificadas como $A$. flavus (47) foram mantidas em tubos contendo o meio Ágar Batata Dextrose (BDA) para avaliação do potencial toxigênico, utilizando a metodologia descrita por Lin; Dianese (1976). As cepas foram mantidas em BDA por dez dias a $25^{\circ} \mathrm{C}$ e, em seguida, um fragmento de cada colônia foi inoculado em uma placa de Petri contendo o meio Ágar Coco, sendo incubada, novamente, por dez dias a $25^{\circ} \mathrm{C}$. Após o crescimento, o conteúdo das placas foi triturado e pesado, e as aflatoxinas foram extraídas com $30 \mathrm{~mL}$ de clorofórmio para cada de $10 \mathrm{~g}$ do cultivo. Após 30 minutos de agitação, o solvente foi filtrado em papel de filtro contendo sulfato de sódio e terra de diatomácea. O filtrado foi evaporado até a secura e transferido para um volume conhecido e aplicado em placa de sílica gel G 60 (Merck), acompanhado de um padrão quantitativo de aflatoxinas $B_{1}, B_{2}, G_{1}$ e $G_{2}$. A placa foi eluída unidimensionalmente 
com a mistura de solventes clorofórmio:acetona $(9: 1 \mathrm{v} / \mathrm{v})$ (Aoac, 1980). Após a secagem do solvente, realizou-se a leitura das amostras e do padrão em fotodensitômetro (CS 9000, Shimadzu). A quantificação foi feita por comparaçẫo das áreas das amostras com as áreas dos padrôes de aflatoxinas.

\section{Ácido ciclopiazônico}

As cepas estudadas foram mantidas em tubos contendo Ágar batata dextrose a $25^{\circ} \mathrm{C}$ por sete dias. Foram inoculados 1,0 $\mathrm{mL}$ de suspensão de esporos, de cada cepa, em frascos contendo $25 \mathrm{~mL}$ de caldo de Czapeck-Dox (DIFCO) e incubados a $25^{\circ} \mathrm{C}$ por 12 dias. As culturas foram filtradas e os caldos foram extraídos duas vezes com $25 \mathrm{~mL}$ de clorofórmio (SANCHEZ et al., 2004). Os extratos foram filtrados, evaporados até a secura e rediluídos com 1,0 mL de metanol grau CLAE. O ácido ciclopiazônico foi quantificado por cromatografia líquida de alta eficiência (CLAE), tendo como fase móvel acetonitrila:tampão acetato de amônio $(0,05 \mathrm{M}, \mathrm{pH} 5)$ $(8: 2 \mathrm{v} / \mathrm{v})$, fluxo de $0,6 \mathrm{~mL} \cdot \mathrm{min}^{-1}$, detector de UV em $284 \mathrm{~nm}$ (Losito et al., 2002), coluna ODS C8 4,6X250 mm de 5 micron (Shimadzu). O método de quantificação das amostras foi padrão externo, utilizando curva de calibraçáo com 5 pontos $(0,611 ; 1,223 ; 2,446 ; 4,892$ e 9,798 mg.mL-1 $)$.

\section{RESULTADOS E DISCUSSÃO}

Cepas de A. flavus produtoras de ACP e aflatoxinas são frequentemente isoladas de amendoim (Luk et al., 1977;
GALLAGHeR et al., 1978; VAAMONDE et al., 2006), indicando a natural ocorrência simultânea dessas toxinas neste substrato. Tendo em vista os danos à saúde humana e animal causados pela ocorrência simultânea das aflatoxinas e do ACP, a produção dessas toxinas em produtos agrícolas deve ser controlada (VAAMONDE et al., 2006).

Neste estudo foram isoladas 47 cepas de A. flavus, sendo 2 nas cascas dos frutos recém-formados; 3 nos grãos e 2 nas cascas no estágio de granação; 5 nos grãos e 1 nas cascas dos frutos maduros; 3 nos grãos e 1 nas cacas dos frutos após a secagem; 12 nas cascas e 18 nos grãos dos frutos armazenados. Das cepas isoladas no campo, $88,2 \%$ foram produtoras de aflatoxinas, $41,7 \%$ foram produtoras de ACP, $35,3 \%$ produziram ambas as toxinas, $52,9 \%$ produziram apenas aflatoxinas, $5,8 \%$ produziram apenas $\mathrm{ACP}$ e $5,8 \%$ não foram produtoras. Já durante o armazenamento, 93,3\% foram produtoras de aflatoxinas, $86,6 \%$ foram produtoras de ACP, 80,0\% produziram ambas as toxinas e $6,6 \%$ produziram apenas ACP. As concentraçóes de aflatoxina $B_{1}$ e $B_{2}$ variam de 18.560 a 30,0 mg.kg-1 e de 220 a 1,0 mg. $\mathrm{kg}^{-1}$, respectivamente, e as de ACP variam de 17,45 a $2,36 \mathrm{mg} \cdot \mathrm{mL}^{-1}$, nos frutos colhidos durante a maturação (Tabela 1). Nas amostras coletadas durante o armazenamento, as concentraçóes de aflatoxinas $B_{1}, B_{2}$ e ACP variaram de 14.128 a $0,10 \mathrm{mg} \cdot \mathrm{kg}^{-1}$, de 420 a $0,19 \mathrm{mg} / \mathrm{kg}$ e de 67,61 a $0,64 \mathrm{mg} \cdot \mathrm{mL}^{-1}$, respectivamente (Tabela 2).

Em média, as cepas isoladas no campo produziram maior quantidade de aflatoxinas $\left(\mathrm{AFB}_{1}=5.309,88\right.$ $\mathrm{mg} \cdot \mathrm{kg}^{-1}$ e $\left.\mathrm{AFB}_{2}=71,64 \mathrm{mg} \cdot \mathrm{kg}^{-1}\right)$ em relação às isoladas

Tabela 1. Concentrações de aflatoxinas $B_{1}, B_{2}\left(\mathrm{mg} \cdot \mathrm{kg}^{-1}\right)$ e ácido ciclopiazônico $\left(\mathrm{mg} \cdot \mathrm{mL}^{-1}\right)$ de cepas de Aspergillus flavus isoladas de amostras de grãos e cascas de amendoim em diferentes estágios de maturação.

\begin{tabular}{|c|c|c|c|c|c|}
\hline $\begin{array}{l}N^{0} \text { da } \\
\text { cepa }\end{array}$ & Estágio de maturação & $\begin{array}{l}\text { Material de } \\
\text { procedência }\end{array}$ & $\mathrm{AFB}_{1}$ & $\mathrm{AFB}_{2}$ & ACP \\
\hline 01 & granação & Grão & ND & ND & ND \\
\hline 02 & granação & Grão & 13.900 & 50 & ND \\
\hline 03 & granação & Grão & ND & ND & 4,48 \\
\hline 04 & maduro & Grão & 18.560 & 150 & 8,07 \\
\hline 05 & maduro & Grão & 4.300 & 220 & ND \\
\hline 06 & maduro & Grão & 13.390 & 140 & 11,16 \\
\hline 07 & maduro & Grão & 870 & 60 & 12,18 \\
\hline 08 & maduro & Grão & 1.011 & 110 & ND \\
\hline 09 & seco & Grão & 600 & 10 & ND \\
\hline 10 & seco & Grão & 270 & 3,00 & 15,34 \\
\hline 11 & seco & Grão & 9.130 & 160 & ND \\
\hline 12 & recém-formado & Casca & 30 & 4,00 & 17,45 \\
\hline 13 & recém-formado & Casca & 190 & 1,00 & ND \\
\hline 14 & granação & Casca & 7.700 & 200 & 2,36 \\
\hline 15 & granação & Casca & 11.460 & 40 & ND \\
\hline 16 & maduro & Casca & 87 & ND & ND \\
\hline 17 & seco & Casca & 8.770 & 70 & ND \\
\hline
\end{tabular}

$\mathrm{AFB}_{1}$ : Aflatoxina $\mathrm{B}_{1}$; $\mathrm{AFB}_{2}$ : Aflatoxina $\mathrm{B}_{2}$; ACP: Ácido Ciclopiazônico; ND: Não Detectado 
Tabela 2. Concentrações de aflatoxinas $B_{1}, B_{2}\left(\mathrm{mg} \mathrm{kg}^{-1}\right)$ e ácido ciclopiazônico (mg. $\mathrm{mL}^{-1}$ ) de cepas de Aspergillus flavus isoladas de amostras de grãos e cascas de amendoim armazenados.

\begin{tabular}{|c|c|c|c|c|}
\hline $\begin{array}{l}\text { No da } \\
\text { cepa }\end{array}$ & $\begin{array}{l}\text { Material de } \\
\text { procedência }\end{array}$ & $\mathrm{AFB}_{1}$ & $\mathrm{AFB}_{2}$ & ACP \\
\hline 01 & Grão & 0,56 & ND & 34,50 \\
\hline 02 & Grão & 0,24 & ND & 23,67 \\
\hline 03 & Grão & 1,92 & 0,4 & 45,54 \\
\hline 04 & Grão & 203,36 & 4,69 & 0,64 \\
\hline 05 & Grão & 11.983 & 107 & 1,82 \\
\hline 06 & Grão & 95,90 & 2,0 & 6,09 \\
\hline 07 & Grão & 4.462 & 10,05 & 40,63 \\
\hline 08 & Grão & 0,58 & ND & ND \\
\hline 09 & Grão & 185,04 & 1,61 & 9,08 \\
\hline 10 & Grão & 246,36 & 1,53 & 21,58 \\
\hline 11 & Grão & 7,29 & 1,5 & 6,34 \\
\hline 12 & Grão & 14.128 & 420 & 14,93 \\
\hline 13 & Grão & 0,20 & ND & 50,64 \\
\hline 14 & Grão & 171,86 & 2,14 & 1,05 \\
\hline 15 & Grão & 2,09 & 0,4 & 56,52 \\
\hline 16 & Grão & ND & ND & 24,49 \\
\hline 17 & Grão & 105,27 & 0,79 & ND \\
\hline 18 & Grão & 209,85 & 1,34 & 33,58 \\
\hline 19 & Casca & 118,82 & 0,99 & 67,41 \\
\hline 20 & Casca & ND & ND & 32,68 \\
\hline 21 & Casca & 37,36 & 1,39 & 31,81 \\
\hline 22 & Casca & 3,00 & 1,50 & 1,10 \\
\hline 23 & Casca & 0,10 & 0,3 & 11,82 \\
\hline 24 & Casca & 76,20 & 0,19 & 29,81 \\
\hline 25 & Casca & 30,52 & 0,9 & ND \\
\hline 26 & Casca & 67,35 & 0,16 & 1,40 \\
\hline 27 & Casca & 12,64 & 0,5 & ND \\
\hline 28 & Casca & 61,43 & 0,39 & 4,49 \\
\hline 29 & Casca & 3.236 & 290 & 4,16 \\
\hline 30 & Casca & 82,63 & 2,02 & 4,83 \\
\hline
\end{tabular}

$\mathrm{AFB}_{1}$ : Aflatoxina $\mathrm{B}_{1}$; $\mathrm{AFB}_{2}$ : Aflatoxina $\mathrm{B}_{2}$; $\mathrm{ACP}$ : Ácido Ciclopiazônico; ND: Não Detectado. no armazenamento $\left(\mathrm{AFB}_{1}=1.182,91 \mathrm{mg} \cdot \mathrm{kg}^{-1}\right.$ e $\mathrm{AFB}_{2}=$ 28,39 mg. $\left.\mathrm{kg}^{-1}\right)$, enquanto a produção de ACP foi maior pelas cepas isoladas no armazenamento $\left(18,68 \mathrm{mg} \cdot \mathrm{mL}^{-1}\right)$ do que no campo $\left(4,17 \mathrm{mg} \cdot \mathrm{mL}^{-1}\right)$, resultados significantes pelo teste ANOVA $(\mathrm{p}<0,05)$. Quando aplicado o teste de Tukey-Kramer, somente considerou-se estatisticamente significante $(\mathrm{p}<0,001)$ a diferença de concentraçôes entre a aflatoxina $\mathrm{B}_{1}$ do campo e do armazenamento. Outros estudos relataram a presença de $A$. flavus em grãos e cascas de amendoim das variedades Runner IAC 886 e Caiapó em diferentes estágios de maturação, e também durante o armazenamento, assim como a presença de aflatoxinas e ACP (Zorzete et al., 2012; 2013). A alta frequência de $A$. flavus e a presença de aflatoxinas foram verificadas em campos de amendoim de diferentes regióes do estado Sáo Paulo (AtAyde et al., 2012).

No Brasil, além de amendoim, há relatos de ACP em produtos derivados de tomates e em leite comercial, coletados no estado de São Paulo (MotTa; SoAres, 2000; 2001; Oliveira et al., 2006).

Os achados de ACP em amendoim e milho indicam que a extensão do potencial de exposição do homem e animais a esta toxina é maior do que se acreditava (Norred, 1987), sendo que o ACP pode ter contribuído com as aflatoxinas, para a "Tukey X Disease" (VAamonde et al., 2003).

\section{CONCLUSÃO}

Cepas de Aspergillus flavus produtoras de aflatoxinas $\mathrm{B}_{1}$ e $\mathrm{B}_{2}$ e de ácido ciclopizônico foram isoladas em grãos de casca de amendoim antes, após e durante a colheita, assim como no armazenamento.

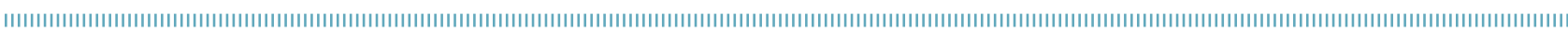

\section{REFERÊNCIAS}

ANDERSON, W.F.; HOLBROOK, C.C.; WILSON, D.M. Development of greenhouse screening for resistance to Aspergillus parasiticus infection and preharvest aflatoxin contamination in peanut. Mycopathologia, New York, v.135, p.115-118, 1996.

ARX, J.A. The genera of fungi sporulating in pure culture. 2. ed. Vaduz: J. Cramer, 1974. 315p.

ATAYDE, D.D.; REIS, T.A.; GODOY, I.J.; ZORZETE, P.; REIS, G.M.; CORREA, B. Mycobiota and aflatoxins in a peanut variety grown in different regions in the state of São Paulo, Brazil. Crop Protection, Londres, v.33, p.7-12, 2012.

ASSOCIATION OF OFFICIAL ANALYTICAL CHEMISTS (AOAC). Official Methods of Analysis. 3. ed. Washington, D.C.: AOAC, 1980.
BAQUIÃO, A.C.;ZORZETE, P.; REIS, T.A.; ASSUNÇÃO, E.; VERGUEIRO, S.; CORREA, B. Mycoflora and mycotoxins in field samples of Brazil nuts. Food Control, Vurrey, v.28, p.224-229, 2012.

BARNETT, H.L.; HUNTER, B.B. Illustrated Genera of Imperfect Fungi. Minneapolis: Burguess Publishing Company, 1965. 232p.

BARRON, G.L. The Genera of Hyphomycetes from soil. New York: Robert E. Krieger Publishing Company, 1972. 364p.

BHATTACHARYA, K.; RAHA, S. Deteriorative changes of maize, groundnut and soybean seeds by fungi in storage. Mycopathologia, New York, v. 155, p. 135-141, 2002.

CALORI-DOMINGUES, M.A.; FONSECA, H. Laboratory evaluation of chemical control of aflatoxin production in unshelled 
peanuts (Arachis hypogaea L.). Food Additives and Contaminants, Londres, v.12, n.3, p.347-350, 1995.

ELLIS, M.B. Dematiaceous Hyphomycetes. Wallinford, Reino Unido: CAB International, 1993. 608p.

GALLAGHER, R.T., RICHARD, J.L., STAHR, H.M., COLE, R.J. Cyclopiazonic acid production by aflatoxigenic and non-aflatoxigenic strains of Aspergillus flavus. Mycopathologia, New York, v.66, p.31-36, 1978

GONÇALEZ, E.; NOGUEIRA, J.H.C.; FONSECA H.; FELICIO, J.D.; PINO, F.A.; CORREAA, B. Mycobiota and mycotoxins in Brazilian peanut kernels from sowing to harvest. International Journal of Food Microbiology, v.123, p.184-190, 2008a.

GONÇALEZ, E.; SOUZA, T.N.; ROSSI, M.H.; FELICIO, J.D.; CORRÊA, B. Avaliação da micoflora e ocorrência de micotoxinas em cascas de amendoim em diferentes estágios de maturação da vagem. Ciência e Agrotecnologia, Lavras, v.32, p. $1380-1386,2008 b$.

GQALENI, N.; SMITH, J.E.; LACEY, J.; GETTINBY, G. Effects of temperature, water activity, and incubation time on production of aflatoxins and cyclopiazonic acid by an isolate of Aspergillus flavus in surface Ágar culture. Applied and Environmental Microbiology, Washington, v.63, n.3, p.1048-1053, 1997.

HORN, B.W.; DORNER, J.W.; GREENE, R.L.; BLANKENSHIP, P.D.; COLE, R.J. Effect of Aspergillus parasiticus soil inocculum on invasion of peanut seeds. Mycopathologia, New York, v.125, p.179-191, 1994.

HORN, B.W.; GREENE, R.L.; DORNER, J.W. Effect of corn and peanut cultivation a soil populations of Aspergillus flavus and Aspergillus parasiticus in Souhwestern Georgia. Applied and Environmental Microbiology, Washington, v.61, n.7, p.247275, 1995.

HORN, B. W.; GREENE, R. L.; SORENSEN, R. B.; BLANKENSHIP, P. D.; DORNER, J. W. Conidial movement of nontoxigenic Aspergillus flavus and Aspergillus parasiticus in peanut fields following application to soil. Mycopathologia, New York, v.151, p.81-92, 2000.

HUSSEIN, S.H.; BRASEL, J.M. Toxicity, metabolism, and impact of mycotoxins on humans and animals. Toxicology, v.167, p. 101-134, 2001.

ISMAIL, M.A. Deterioration and spoilage of peanuts and desiccated coconuts from two sub-saharan tropical East African countries due to the associated mycobiota and their degradative enzymes. Mycopathologia, New York, v. 150, p.67-80, 2000.

LISKER, N.; MICHAELI, R.; FRANK, Z.R. Mycotoxigenic potential of Aspergillus flavus strains isolated from groundnuts growing in Israel. Mycopathologia, New York, v.122, p.177-183, 1993.

LOSITO, I., et al. LC-ion trap eletrospray MS-MS for determination of cyclopiazonic acid in milk samples. Analyst, Londres, v.127, n.4, p.499-502, 2002.
LUK, K.C., KOBBE, B., TOWNSEND, J.M. Production of cyclopiazonic acid by Aspergillus flavus link. Applied and Environmental Microbiology, Washington, v.33, p.211-212, 1977.

MOSS, M.O. Mycotoxic fungi. In: ELEY, A.R. (Ed.). Microbial Food Poisoning. 2. ed. Londres: Chapmen, 1996. p.75-93.

MPHANE, F.A.; SIAME, B.A.; TAYLOR, J.E. Fungi, aflatoxin and cyclopiazonic acid associated with peanut retailing in Botswana. Journal Food Protection, Des Moines, v.67, n.1, p.96-1002, 2004.

NAKAI, V.K.; ROCHA, L.O.; GONÇALEZ, E.; FONSECA, H.; ORTEGA, E.M.M.; CORRÊA, B. Distribution of fungi and aflatoxin in a stored peanut variety. Food Chemistry, Barking, v.106, p.285-290, 2008.

NELSON, P.E.; TOUSON, T.A.; MARASAS, W.F.O. Fusarium species. An illustrated manual for identification. Pennsylvania: University Press, 1983. 193p.

OLIVEIRA, C.A.; ROSMANINHO, J.; ROSIM, R. Aflatoxin M 1 and cyclopiazonic acid in fluid milk traded in São Paulo, Brazil. Food Additives and Contaminants, Londres, v.23, n.2, p.196-201, 2006.

PILDAIN, M.B.; VAAMONDE, G.; CABRAL, D. Analysis of population structure of Aspergillus flavus peanut based on vegetative compatibility, geographic origin, mycotoxin and sclerotia production. International Journal Food Microbiology, v.93, p.31-40, 2004.

PITT, J.I.; KING, A.D.; HOCKING, A.D. Dicloran-Rose Bengal Medium for enumeration and isolation of molds from foods. Applied and Environmental Microbiology, Washington, v.37, p.959-970, 1979.

RAPER, K.B.; FENNEL, D.I. The genus Aspergillus. Baltimore: Willians Wilkins, 1965. 686p.

RIDELL, R.W. Permanent stained mycological preparations obtained by slid culture. Mycologia, New York, v.42, p.265270, 1950

RUDREW, S.; CRAFT, J.; AIDOO, K. Occurrence of toxigenic Aspergillus spp. and aflatoxins in selected food commodities of Asian origin in the west of Scotland. Food and Chemical Toxicology, Oxford, v.55, p.653-658, 2013.

SANCHEZ, E.; HEREDIA, AN.; GARCIA, S. Inhibition of growth and myctoxins production of Aspergillus flavus and Aspergillus parasiticus by extracts of Agave species. International Journal Food Microbiology, v.98, p.271-278, 2004.

SIAME, B.A.; MPUCHANE, S.F.; GASHE, B.A.; ATTLEY, J.; TEFFERA, G. Occurrence of aflatoxins, fumonisin $B_{1}$ and zearalenone in foods and feeds in Botswana. Journal of Food Protection, Des Moines, v.61, n.12, p.1670-1673, 1998.

SILKER, J.H.; ELLIOT, R.P. Ecologia Microbiana de los Alimentos. Acribia, Zaragoza, v. 1, p.74-96, 1980. 
VAAMONDE, G.; PATRIARCA, A.; PINTO, V.F.; COMERIO, R.; DEGROSSI, C. Variability of aflatoxin and cyclopiazonic acid production by Aspergillus section flavi from different substrates in Argentina. International Journal Food Microbiology, v.88, p.79-84, 2003.

VAAMONDE, G.; PATRIARCA, A.; PINTO, V.F. Effects of water activity and temperature on production of aflatoxin and cyclopiazonic acid by Aspergillus flavus in peanuts. Advances in Experimental Medicine and Biology, New York, v.571, p.225-235, 2006.
ZORZETE, P; REIS, T.A.; FELICIO, J.D.; BAQUIÃO, A.C.; MAKIMOTO, P.; CORREA, B. Fungi, mycotoxins and phyoalexin in peanut varieties, during plant growth in the field. Food Chemistry, Barking, v.129, p.957-964, 2011.

ZORZETE, P; BAQUIÃO, A.C.; ATAYDE, D.D.; REIS, T.A.; GONÇALEZ, E.; CORREA, B. Mycobiota, aflatoxins and cyclopiazonic acid in stored peanut cultivars. Food Research International, Essex, v.52, p.380-386, 2013. 